\title{
Activation of right parietal cortex during memory retrieval of nonlinguistic auditory stimuli
}

\author{
ELLen C. KLOSTERManN \\ University of California, Berkeley, California \\ Psyche Loui \\ Beth Israel Deaconess Medical Center and Harvard Medical School, Cambridge, Massachusetts \\ AND \\ ArThur P. Shimamura \\ University of California, Berkeley, California
}

\begin{abstract}
In neuroimaging studies, the left ventral posterior parietal cortex (PPC) is particularly active during memory retrieval. However, most studies have used verbal or verbalizable stimuli. We investigated neural activations associated with the retrieval of short, agrammatical music stimuli (Blackwood, 2004), which have been largely associated with right hemisphere processing. At study, participants listened to music stimuli and rated them on pleasantness. At test, participants made old/new recognition judgments with high/low confidence ratings. Right, but not left, ventral PPC activity was observed during the retrieval of these music stimuli. Thus, rather than indicating a special status of left PPC in retrieval, both right and left ventral PPC participate in memory retrieval, depending on the type of information that is to be remembered.
\end{abstract}

Retrieval-related activity in the parietal cortex is consistently observed in human neuroimaging studies (for reviews, see Cabeza, Ciaramelli, Olson, \& Moscovitch, 2008; Vilberg \& Rugg, 2008; Wagner, Shannon, Kahn, $\&$ Buckner, 2005). This finding centers on the successful retrieval effect, in which activity in the posterior parietal cortex (PPC) during memory retrieval is greater for items recognized as old than for those recognized as new. This effect has been observed over a wide range of stimuli and task demands and is associated with activity in the dorsal PPC (superior parietal lobule/intraparietal sulcus, or IPS), ventral PPC (inferior parietal lobule/supramarginal and angular gyri/BA 39 and BA 40), and medial PPC (precuneus).

Recent meta-analyses have suggested that a functional dissociation exists in the contribution of dorsal and ventral PPC regions with respect to two memory retrieval processes-recollection and familiarity (Cabeza et al., 2008; Vilberg \& Rugg, 2008). Recollection refers to a specific memory of the spatial-temporal context of a remembered event or stimulus, whereas familiarity is driven by a more general sense of knowing. Familiarity judgments have been associated with dorsal PPC regions along the IPS, although some have suggested that activity in these regions reflects more general cognitive processes involved in both familiarity and recollection, such as indexing the salience of a stimulus (see Vilberg \& Rugg, 2008). Recollection judgments have been associated with ventral PPC regions, particularly in areas lateral and inferior to the IPS (BA 39).

A prominent finding from these studies is that the left ventral PPC is particularly involved in mediating recollection-related effects. Although some studies have shown bilateral activation (e.g., Shannon \& Buckner, 2004), activity in the left ventral PPC has always been greater than activity in the right PPC (Rugg \& Henson, 2002). Despite the consistency of the lateralized finding, the factors that mediate it have not been well studied. Some studies have attempted to use different stimulus types to address the effect. Schloerscheidt and Rugg (1997) found that event-related potentials (ERPs) indexed a successful retrieval effect that occurred in the left hemisphere for both words and pictures of objects, despite previous findings (Smith, 1989) showing that medial temporal lobe (MTL) responses for these two stimulus types are lateralized. Shannon and Buckner showed that PPC activity for natural sounds (e.g., the sound of a dog barking) is left-lateralized, and that this activity is more lateralized than that observed for picture stimuli (e.g., a picture of a dog). One concern, however, is that these stimuli, and indeed practically all previous stimuli, were either verbal in nature or could be easily described verbally (e.g., words, pictures, natural sounds).

Recently, Guerin and Miller (2009) directly addressed the role of verbal mediation in memory retrieval by com- 
paring fMRI activity in the ventral PPC during the recollection of words and unfamiliar faces. It was argued that unfamiliar faces are complex visual stimuli that would not depend significantly on verbal processes. Left-lateralized ventral PPC activity was observed for both word and unfamiliar face stimuli, suggesting a specialized role for the left ventral PPC in recollection. However, it is not uncommon to describe unfamiliar face stimuli according to their outstanding facial features (e.g., having "a long nose" or "bushy eyebrows"), especially if participants intend to remember them. Thus, whereas verbal encoding of unfamiliar faces is not necessary, such stimuli may still engage left-lateralized verbal processes.

In the present study, we assessed the successful retrieval effect, using short clips of agrammatical music stimuli. These stimuli were taken from novel musical compositions (Blackwood, 2004) and consist of synthesized timbres and harmonies (e.g., Audio File S1). Thus, they are extremely difficult to verbalize, since they do not adhere to known scales or standard grammatical systems used in musical compositions around the world. It is important that these music stimuli are preexperimentally unfamiliar and not associated with lyrics, since the presence of these characteristics might lead participants to rely on verbal, left-hemisphere-mediated processing.

Previous studies have shown that the right hemisphere is preferentially involved in the perception and encoding of musical stimuli (Zatorre, Belin, \& Penhune, 2002). Additionally, impairment in the recognition of unfamiliar melodies occurs following right, but not left, temporal lobectomy, suggesting that memory for musical stimuli depends more on the right than on the left hemisphere (Samson \& Zatorre, 1991). Thus, these stimuli, which were preexperimentally unfamiliar and include extremely novel perceptual features, offer a strong test of the role of the left PPC in mediating the successful retrieval effect. If the left ventral PPC is always preferentially engaged during successful retrieval, it should be active in the present experiment, despite the fact that these stimuli are encoded and processed in the right hemisphere. However, if retrieval-related activation is linked to brain regions involved in the processing of the stimuli, we expect the right ventral PPC to be active, but not the left.

\section{METHOD}

\section{Participants}

Sixteen volunteers from the University of California, Berkeley community ( 9 females; average age, 22.4 years) participated in this study. All of the participants were right-handed, native English speakers. To ensure that participants would have no previous exposure to these novel musical stimuli, participants were limited to nonmusicians (i.e., individuals with no musical training who reported that they were unable to play an instrument and were unable to read sheet music). Participants gave informed consent according to the procedures of the University of California, Berkeley, and were paid for their participation.

\section{Stimuli and Behavioral Procedure}

The stimuli were short musical clips that involved atypical timbres and harmonies; 110 music clips were cropped from a recording of novel compositions (Blackwood, 2004). Clips from this recording of novel compositions were chosen as stimuli because they were nonverbalizable, did not adhere to the traditional rules of musical structure, and were unfamiliar to all participants. Clips ranged from 1,800 to $2,000 \mathrm{msec}$ long and were normalized in loudness. The stimuli were randomly assigned to one of two study-test sets. In one study-test set, participants learned 27 music clips and were tested with 54 clips ( 27 old, 27 new). In the second set, participants learned 28 music clips and were presented with 56 clips during the test phase ( 28 old, 28 new). The order of the study-test sets was counterbalanced across participants. During both study and test phases, participants had their eyes closed. To reduce extraneous noise from the scanner, electrodynamic noise-suppression headphones (MR-Confon, Magdeburg, Germany) were used to present the music stimuli.

A study trial consisted of a music clip presentation $(\sim 1,800$ to $2,000 \mathrm{msec}$ ) and intertrial intervals (ITIs) that varied from 4.4 to $8.8 \mathrm{sec}$ (jittered for event-related fMRI). Participants were told to remember these music clips for a later memory test. For each music clip, participants made a pleasant or unpleasant judgment, using keypress responses. The buttons used to make pleasant or unpleasant responses were counterbalanced across participants. To facilitate learning of the study set, the same stimuli were presented a second time in a different random order.

During the test phase, participants listened to a random series of old and new music clips and were tested on the stimuli only once. For each test clip, participants made old/new recognition judgments with high/low confidence ratings. Specifically, they were instructed to give an "old-high" rating if they were $100 \%$ certain that they heard the music clip during the study phase, an "old-low" rating if they thought the item was old but were not certain, a "new-high" rating if they were $100 \%$ certain they had not heard the music clip during the study phase, or a "new-low" rating if they thought the item was new but were not certain. The music clips were presented for 1,800-2,000 msec, with a 4.4- to 8.8-sec jittered ITI in two test phase runs. The buttons used to make responses were counterbalanced across participants. For both study and test phases, E-Prime software (Psychology Software Tools, Inc., Pittsburgh, PA; www .pstnet.com) was used to present stimuli and collect responses.

\section{fMRI Methods}

Participants were scanned in a $4 \mathrm{~T}$ Varian INOVA scanner (Varian Inc., Palo Alto, CA) using a two-shot gradient echo, echoplanar imaging (EPI) sequence $(\mathrm{TR}=2.2 \mathrm{sec}, \mathrm{TE}=28 \mathrm{msec}$, flip angle $20^{\circ}, 64 \times 66$ matrix, FOV $=22.4 \mathrm{~cm}^{2}$ ). For each volume, twenty $3.5-\mathrm{mm}$-thick slices (slice gap $=0.5 \mathrm{~mm}$ ) were obtained. Each scanning session consisted of eight runs - four study phase runs and four test phase runs. Each run began with 10 dummy RF scans to allow time for steady state tissue magnetization and to minimize the effects of head movements that might occur at the onset of the scanner noise.

A gradient-echo multislice (GEMS) sequence was used to acquire high-resolution T1-weighted anatomical scans $(3.50 \times 0.875 \times$ $0.875 \mathrm{~mm}$ ) using the same 20 slices defined for the EPI scans. So that participants could be normalized to the Montreal Neurological Institute (MNI) atlas space for group analyses, we also acquired 3-D T1-weighted magnetization-prepared fast low-angle shot (MPFLASH) scans for each participant.

\section{fMRI Analysis}

The data were analyzed using SPM2 software (Wellcome Department of Cognitive Neurology, London). The data were first reconstructed into SPM2 image files and interpolated to a TR of 1.1 using a linear time interpolation algorithm, doubling the effective sampling rate (Noll, Stenger, Vazquez, \& Peltier, 1999). Functional and anatomical images were then recalibrated, such that the origin of all images was fixed to the anterior commissure. The functional images were then realigned, using the first functional image acquired as the reference. A general linear model was run using the six conditions of interest during retrieval (old-high, old-low, new-high, 
Table 1

Accuracy, Reaction Time (RT), and Proportions of Correct High- and Low-Confidence Hits and Correct Rejections During the Test Phase

\begin{tabular}{lrr} 
& \multicolumn{1}{c}{$M$} & \multicolumn{1}{c}{$S D$} \\
\hline & Accuracy & \\
New-high & 81.02 & 12.35 \\
New-low & 65.88 & 6.48 \\
Old-high & 85.40 & 10.37 \\
Old-low & 58.63 & 14.58 \\
MS & 25.68 & 9.48 \\
FA & 25.34 & 6.98 \\
NR & 10.85 & 13.62 \\
& RT (msec) & \\
New-high & 696.71 & 205.81 \\
New-low & 943.95 & 285.56 \\
Old-high & 876.42 & 251.68 \\
Old-low & 649.47 & 175.14 \\
MS & 903.15 & 304.32 \\
FA & 820.72 & 274.10 \\
& Proportion & \\
New-high & .26 & .13 \\
New-low & .34 & .12 \\
Old-high & .32 & .17 \\
Old-low & .24 & .14 \\
\hline
\end{tabular}

Note- High, high confidence; Low, low confidence; MS, miss; FA, false alarm; NR, nonresponse.

new-low, false alarms, and misses) and two conditions of interest during encoding (items later remembered and items later forgotten) as regressors, and the experimental contrasts were identified. Each participant's anatomical images were coregistered. The experimental contrast files were normalized onto a standard brain volume. Group statistics ( $t$ tests) were then performed on the experimental contrasts using a threshold of $p<.0025$, uncorrected.

\section{ROI and Time Course Analysis}

A region of interest (ROI) analysis of the activity in the left ventral PPC was performed. Using the MarsBaR toolbox (Brett, Anton, Valabregue, \& Poline, 2002), we defined two sets of ROIs for the "old-high" versus "new-high" contrast and one set of ROIs for the "old" versus "new" contrast. Finite impulse response time course data were extracted from one set of right and left PPC ROIs for the "old-high" versus "new-high" contrast using MarsBaR.

\section{RESULTS}

\section{Behavioral Results}

Recognition performance is shown in Table 1. Participants' accuracy for high-confidence old items was $85.40 \%(S D=10.37)$. The accuracy for high-confidence new items was $81.02 \%(S D=12.35)$. Participants were significantly more accurate in their high-confidence responses to old and new items than in their low-confidence responses to them $(p \mathrm{~s}<.001)$. The average nonresponse rate across the test phase was $10.85 \%$. This high rate indicates how difficult the task was because of the unusual and unfamiliar nature of the stimuli.

The average reaction time (RT) for high-confidence hits $(M=876.42 \mathrm{msec} ; S D=251.68)$ was significantly slower than the average RT for low-confidence hits $(M=$ $649.47 \mathrm{msec} ; S D=175.14)[t(15)=-4.71, p<.0003]$. However, the average RT for high-confidence correct rejections $(M=696.71 \mathrm{msec} ; S D=205.81)$ was signifi- cantly faster than that for low-confidence correct rejections $(M=943.95 \mathrm{msec} ; S D=285.56)[t(15)=4.42$, $p<.0005]$.

\section{Encoding Phase fMRI Results}

Memory-related activity during encoding was assessed by comparing activity for items subsequently remembered with activity for items later forgotten, regardless of confidence (the Dm effect; see Paller \& Wagner, 2002). Areas significantly more active at encoding for items later remembered, compared with those later forgotten, included right superior temporal cortex $(x=52, y=-8, z=0)$, right PPC ( $x=42, y=-44, z=56)$, left PPC ( $x=-38$, $y=-52, z=56)$, left precuneus, and multiple bilateral frontal regions (see Table 2).

\section{Retrieval Phase fMRI Results}

During the retrieval phase, a univariate comparison of correct "old-high" versus "new-high" items (highconfidence hits vs. high-confidence correct rejections) revealed significant activation in the right ventral PPC $(x=54, y=-56, z=34)$ (see Figure 1). This righthemisphere PPC effect remained significant when a small volume correction analysis was performed using a $10-\mathrm{mm}$ sphere around the right hemisphere coordinates related to recollection in Vilberg and Rugg (2008) $(x=53, y=$ $-61, z=25)$. No significant successful retrieval effect was observed in the left PPC. Even when a more lenient statistical threshold was used for the whole-brain analysis $(p<.05)$, no significant activity in the left ventral PPC was observed. Interestingly, this laterality effect was not observed for medial parietal regions; significant activation was found in bilateral precuneus (for a similar effect, see Guerin \& Miller, 2009). Additional activation was observed in multiple regions within the bilateral frontal lobes (see Table 3).

In an ROI analysis, we examined the "old-high" versus "new-high" contrast in the left hemisphere in more detail. One ROI defined in the left ventral PPC contained

Table 2

Regions of Activity for the Later Successfully Retrieved Versus Later Forgotten Contrast During the Encoding Phase

\begin{tabular}{lrrrrr}
\hline & \multicolumn{5}{c}{ MNI Coordinates } \\
\cline { 2 - 6 } \multicolumn{1}{c}{ Brain Region } & BA & $X$ & \multicolumn{1}{c}{$Y$} & $Z$ & $T$ Score \\
\hline Lt. inferior frontal & 45 & -38 & 38 & 4 & 4.86 \\
Rt. superior temporal & - & 52 & -8 & 0 & 4.09 \\
Rt. medial frontal & 48 & 34 & 18 & 20 & 3.84 \\
Rt. middle frontal & 9 & 48 & 12 & 52 & 3.77 \\
Rt. inferior frontal & 46 & 40 & 34 & 28 & 3.72 \\
Lt. inferior parietal & 40 & -38 & -52 & 56 & 3.66 \\
Lt. postcentral & 3 & -44 & -20 & 38 & 3.64 \\
Rt. superior temporal & 48 & 48 & -20 & 4 & 3.61 \\
Rt. inferior parietal & 40 & 42 & -44 & 56 & 3.52 \\
Lt. precuneus & - & -14 & -72 & 62 & 3.47 \\
Lt. inferior frontal & 48 & 38 & 18 & 24 & 3.45 \\
Rt. inferior frontal & 45 & -48 & 38 & 6 & 3.43 \\
Lt. middle frontal & 46 & -32 & 54 & 16 & 3.41 \\
Rt. superior parietal & - & 24 & -44 & 76 & 3.38 \\
Rt. superior temporal & 48 & 44 & -16 & -6 & 3.38 \\
Rt. middle frontal & 46 & 36 & 20 & 42 & 3.33 \\
\hline
\end{tabular}

Note-Lt., left; Rt., right. 

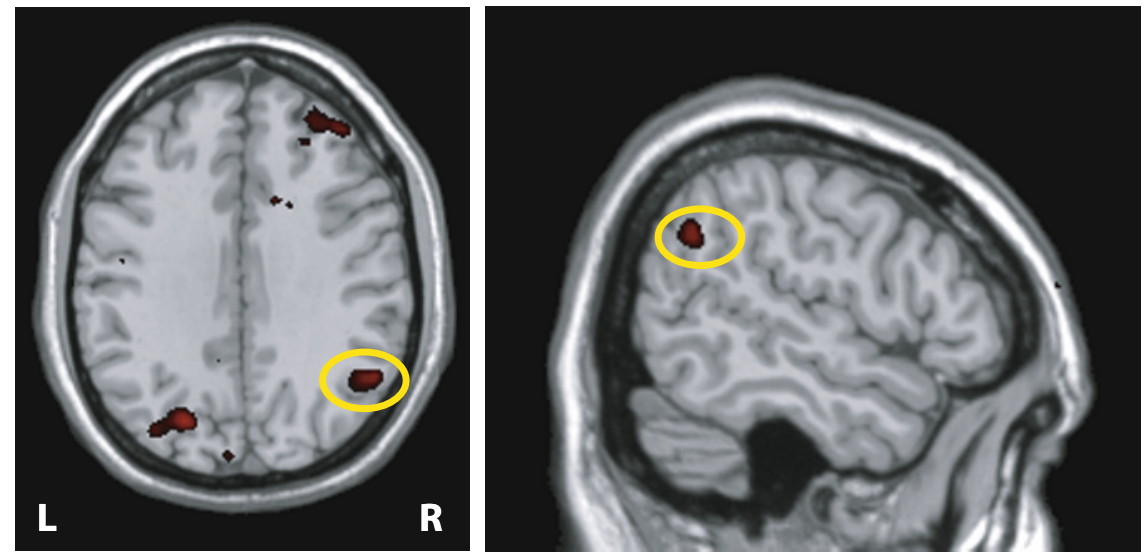

Figure 1. Regions of activity for the high-confidence hits versus high-confidence correct rejections contrast, showing activity in the right, but not left, posterior parietal cortex (BA 39; $p<.0025)$.

voxels corresponding to those active in the right ventral PPC for the "old-high" versus "new-high" contrast ( $p=$ $.05)$. No marginal level of activation in this left PPC region was observed $(p=.62)$. A second ROI consisted of a $10-\mathrm{mm}$ sphere around coordinates in the left PPC that corresponded to peak coordinates of activity in the right PPC for the "old-high" versus "new-high" contrast (see Table 3). Similarly, no significant activity was observed in this left PPC ROI $(p=.66)$.

When the data were collapsed across confidence ratings, a similar effect was observed in the PPC - there was significant activity in the right $\mathrm{PPC}(x=40, y=-60, z=$ $28)$, but not in the left PPC. Furthermore, ROI analyses using a $10-\mathrm{mm}$ sphere centered in the left PPC $(x=-40$, $y=-60, z=28$ ), which corresponded to peak coordinates of activity observed in the right PPC in the "old" versus "new" contrast, were not significant ( $p=.30)$.

Time course data extracted from the right and left hemisphere ROIs (defined as the significant activity in the right PPC for the "old-high" versus "new-high" contrast at $p=$ .05 and the homologous voxels in the left hemisphere) are shown in Figures 2A and 2B. An "old-high" versus "newhigh" difference was observed in the time course data for the right-hemisphere ROI, but not for the left. Importantly, there was a significant interaction between the mnemonic status of the items (old-high vs. new-high) and hemisphere (left vs. right; $p=.007$ ).

\section{DISCUSSION}

Whereas previous research has shown that the successful retrieval effect occurs predominantly in the left ventral PPC (Guerin \& Miller, 2009; Vilberg \& Rugg, 2008), we observed right ventral PPC activity during the retrieval of agrammatical music stimuli. Indeed, there was absolutely no evidence that the effect was bilateral, as has been found for other stimuli (e.g., pictures). Importantly, unlike previous studies that have shown an old-high versus new-high effect in the posterior IPS within BA 19 (for reviews, see Ciaramelli, Grady, \& Moscovitch, 2008; Vilberg \& Rugg,
2008), the left BA 19 activations in this study were located entirely within superior and middle occipital areas.

No study, to our knowledge, has shown a successful retrieval effect in only the right hemisphere. Our finding suggests that the laterality of this effect is dependent on the hemisphere involved in processing the stimuli. We suggest that previous findings of a left-sided distribution of the successful retrieval effect occurred because the stimuli used in these experiments were either verbal (i.e., words) or verbalizable (i.e., natural sounds and faces). This effect could not be attributed to the use of auditory stimuli, given that previous findings demonstrated left-lateralized PPC effects for auditorily presented words and natural sounds (Klostermann, Kane, \& Shimamura, 2008; Shannon \& Buckner, 2004). In our study, bilateral effects were observed in medial parietal regions such as the precuneus. This finding is consistent with other reports of a bilateral effect in this region, and suggests that the medial parietal cortex may play

Table 3

Regions of Activity for the High-Confidence Hits Versus High-Confidence Correct Rejections Contrast During Retrieval

\begin{tabular}{|c|c|c|c|c|c|}
\hline \multirow[b]{2}{*}{ Brain Region } & \multicolumn{5}{|c|}{ MNI Coordinates } \\
\hline & $\mathrm{BA}$ & $X$ & $Y$ & $Z$ & $T$ Score \\
\hline Lt. superior parietal & 5 & -16 & -50 & 64 & 5.30 \\
\hline Lt. superior frontal & 46 & -20 & 54 & 22 & 4.95 \\
\hline Lt. inferior frontal & 44 & -50 & 14 & 16 & 4.92 \\
\hline Lt. middle frontal & - & -42 & 60 & -2 & 4.78 \\
\hline Lt. precuneus & 5 & -10 & -60 & 60 & 4.21 \\
\hline Lt. superior occipital & 19 & -22 & -74 & 34 & 4.01 \\
\hline Rt. middle frontal & 46 & 32 & 44 & 30 & 3.99 \\
\hline Rt. supp. motor area & 6 & 14 & 6 & 62 & 3.80 \\
\hline Rt. precentral gyrus & $44 / 6$ & 36 & 2 & 32 & 3.78 \\
\hline Lt. supp. motor area & 6 & -6 & 8 & 68 & 3.63 \\
\hline Lt. precuneus & 7 & -12 & -66 & 70 & 3.61 \\
\hline Rt. angular gyrus & 39 & 54 & -56 & 34 & 3.55 \\
\hline Lt. caudate & - & -12 & 20 & 8 & 3.52 \\
\hline Rt. middle frontal & 10 & 38 & 52 & 2 & 3.50 \\
\hline Rt. precuneus & 5 & 12 & -50 & 56 & 3.47 \\
\hline Rt. middle frontal & 48 & 42 & 28 & 18 & 3.41 \\
\hline Lt. superior frontal & 11 & -22 & 60 & -4 & 3.31 \\
\hline
\end{tabular}

Note-Lt., left; Rt., right; supp., supplemental. 


\section{A Right Hemisphere}

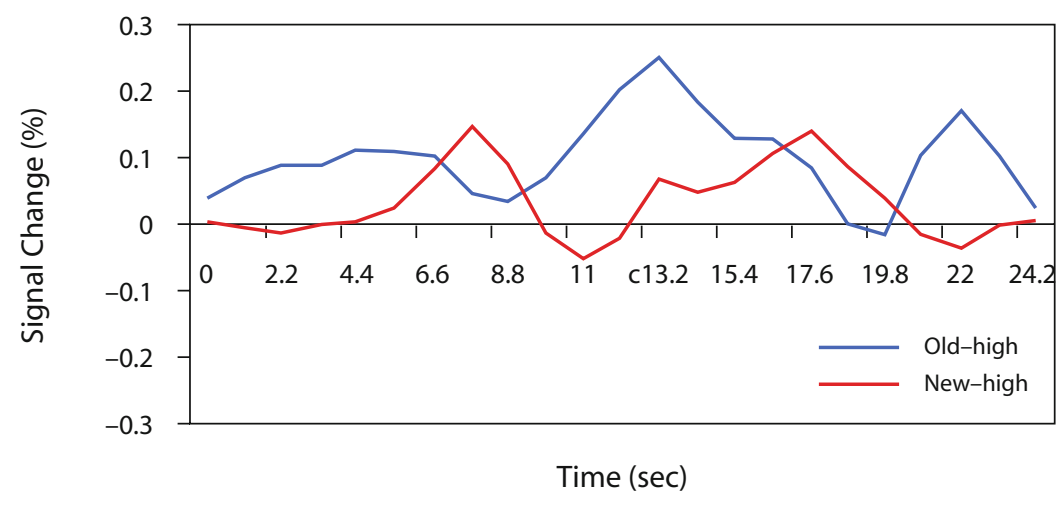

B Left Hemisphere

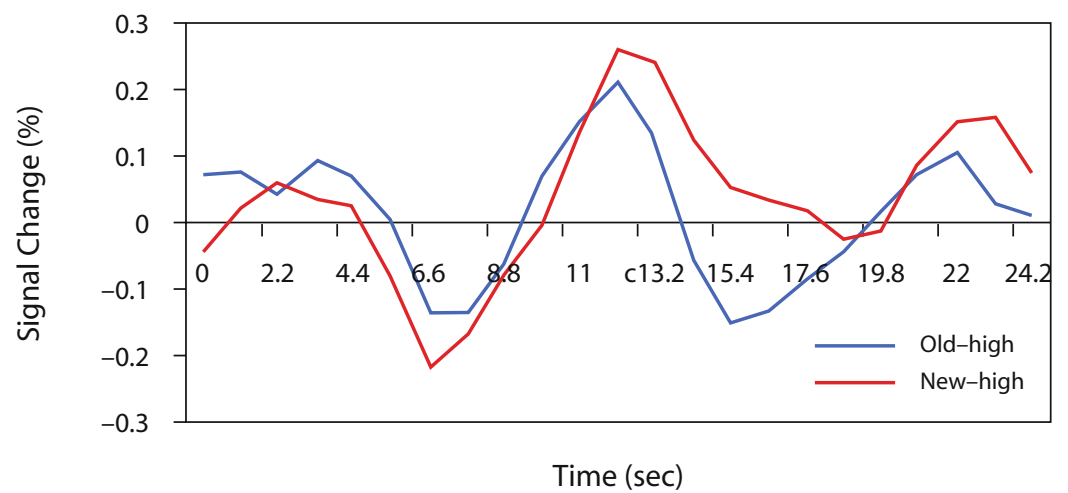

Figure 2. Time course data for the "old-high" versus "new-high" contrast extracted from (A) right and (B) left PPC ROIs.

a decidedly different or indirect role in memory retrieval (Guerin \& Miller, 2009; Vilberg \& Rugg, 2008).

As shown in the time course analysis (Figure 2A), the right PPC response in comparisons of old-high and newhigh judgments occurred rather late, compared with previous effects observed for verbal stimuli (e.g., Henson, Rugg, Shallice, \& Dolan, 2000). This difference may be caused by the unusual nature of the auditory stimuli used in this experiment. We used unfamiliar musical stimuli that depended on sequential processing. Most previous studies have used familiar stimuli (words, pictures), which can be processed quickly. The facility of processing familiar stimuli may result in information arriving at higher level processing areas (PPC, PFC) more quickly than the kind of stimuli used here (Breznitz \& Misra, 2003). Thus, it is likely that the rather late onset of the old/new effect in this experiment occurred because the musical stimuli in this experiment were unfamiliar and had to be processed sequentially.

In the time course analysis for the left PPC, responses were elicited for both hits and correct rejections. Thus, this region was not sensitive to retrieval success, but appeared to respond whenever participants attempted to retrieve information. Previous research has shown that the PPC is active when participants selectively attend to the retrieval of a particular type of mnemonic information (e.g., source information), whether or not they later make a successful judgment about the information retrieved (referred to as the recollection-orienting effect; Dobbins, Rice, Wagner, $\&$ Schacter, 2003; Wagner et al., 2005). Our findings of left PPC activity for both hits and correct rejections (Figure 2B) may reflect this kind of retrieval-orienting process. This orienting process may not have been observed in previous studies because it may have been difficult to distinguish it from the successful retrieval effect usually observed in the left hemisphere.

Our findings from the subsequent memory analysis suggested that greater activity in the right superior temporal lobe and bilateral PPC during encoding is related to better memory retrieval at test. In a recent meta-analysis of episodic encoding, Uncapher and Wagner (2009) identified differences between the encoding functions of the dorsal and ventral PPC. Activity in the dorsal PPC (the superior parietal lobule and intraparietal sulcus) during encoding was related to later successful retrieval, whereas activity in the ventral PPC (intraparietal lobule and temporal-parietal junction) was related to later memory failure. Interestingly, when subsequent memory effects were segregated according to whether the retention interval was long ( $24 \mathrm{~h}$ or longer) or short (45 min or less), all of the studies that 
showed a positive subsequent memory effect in the ventral PPC (in contrast to the overall effect) were those that fell into the short-interval category. In this study, memory was tested within a short retention interval (less than $45 \mathrm{~min}$ ). The bilateral PPC effects in the ventral PPC in our subsequent memory contrast, therefore, fit with Uncapher and Wagner's findings that the ventral PPC was associated with encoding success over short intervals.

Interestingly, in our experiment participants' responses to high-confidence old items were significantly slower than those for low-confidence old items. Most previous studies have shown that RTs to high-confidence old items are faster than are those for low-confidence old items (e.g., Henson et al., 2000). The atypical pattern of RT data observed in this experiment may be caused by the unusual nature of our stimuli. Specifically, participants may have hesitated when making high-confidence responses, given that the stimuli were relatively difficult to learn. This hesitation may have slowed their RTs for this category of response.

Two recent reviews of PPC function have hypothesized about the role of the ventral PPC in successful retrieval. Vilberg and Rugg (2008) have suggested that ventral PPC activity during successful retrieval reflects the engagement of episodic buffers, components of working memory limited in capacity, that temporarily store multimodal, mnemonic information. In contrast, Cabeza et al. (2008) related activity in the ventral PPC to bottom-up attention to memory - the capture of attentional resources by cues or by relevant items spontaneously retrieved by the MTL. Although not meant to directly test either of these theories, the data presented here may inform the validity of these hypotheses. The results of this experiment suggest that the laterality of the PPC effect is dependent on the hemisphere predominantly involved in processing the stimuli. This data does not support the assertion that the PPC is serving the role of an episodic buffer during retrieval, since the lateralization of the activity in a region serving as a multimodal buffer should not be affected by the type of stimuli used. If the left PPC successful retrieval effect reflects activity related to a multimodal episodic buffer, the type of stimulus used should not have affected the laterality of the PPC effect, and the successful retrieval effect should have been observed in the left hemisphere, regardless of the type of stimuli used. This data might, however, fit with Cabeza et al.'s theory that ventral PPC activity reflects bottom-up attention toward relevant information retrieved by the MTL. The right hemisphere is known to be preferentially involved in the perception and encoding of musical stimuli (Zatorre et al., 2002). It is possible, therefore, that the right-sided PPC effect observed in this study reflects bottom-up attention toward music stimuli retrieved by the right MTL in this study. Other theories of PPC function might also predict the laterality effects observed in this experiment. For example, it is possible that the PPC is involved in cortically binding together or integrating episodic features. Thus, during retrieval, PPC and MTL regions may work together in facilitating the reinstatement of episodic features during retrieval. Further experiments should be done to address how the lateral- ity of the successful effect is related to the laterality of the retrieval-related response in the MTL. For example, functional connectivity analyses of this data, and similar studies done using verbal stimuli, might find that the right PPC is functionally correlated with the right MTL during retrieval of music stimuli, whereas the left PPC is functionally correlated with the left MTL during retrieval of verbal stimuli, supporting the bottom-up attention-tomemory hypothesis of PPC functioning.

Although previous neuroimaging investigations have implicated the left PPC during successful retrieval, there is conflicting evidence from neuropsychological studies that have assessed retrieval dysfunction in patients with PPC lesions. Some studies have found no memory impairment in PPC patients (Haramati, Soroker, Dudai, \& Levy, 2008; Simons et al., 2008), whereas others have shown that these patients have impairments in the recollection of autobiographical details (Berryhill, Phuong, Picasso, Cabeza, \& Olson, 2007). Yet, the only report of memory impairments in PPC patients involved patients with bilateral PPC lesions. Our finding of a right PPC retrieval effect, along with previous findings showing left PPC effects (Klostermann et al., 2008), suggests that both the left and right PPC are potentially involved in memory retrieval. Thus, retrieval impairments may occur only in patients with bilateral PPC lesions. Future neuropsychological and neuroimaging studies are required to further delineate the specific PPC regions associated with directed retrieval processes and the manner in which these brain regions participate in a distributed neocortical network.

\section{AUTHOR NOTE}

This research was supported by NSF Grant BCS-0745835 and NIH Grant NS040813 to A.P.S. Correspondence concerning this article should be addressed to E. C. Klostermann, Department of Psychology, Room 3210, Tolman Hall \#1650, University of California, Berkeley, CA 94720-1650 (e-mail: eklostermann@berkeley.edu).

\section{REFERENCES}

Berryhill, M. E., Phuong, L., Picasso, L., Cabeza, R., \& Olson, I. R. (2007). Parietal lobe and episodic memory: Bilateral damage causes impaired free recall of autobiographical memory. Journal of Neuroscience, 27, 14415-14423. doi:10.1523/JNEUROSCI.4163-07.2007

BLACKWOOD, E. (2004). Twelve microtonal etudes for electronic music media, op. 28. Cedille Records CDR 90000018.

Brett, M., Anton, J., Valabregue, R., \& Poline, J. (2002, June). Region of interest analysis using an SPM toolbox [Abstract]. Presented at the 8th International Conference on Functional Mapping of the Human Brain, Sendai, Japan.

Breznitz, Z., \& Misra, M. (2003). Speed of processing of the visualorthographic and auditory-phonological systems in adult dyslexics: The contribution of "asynchrony" to word recognition deficits. Brain \& Language, 85, 486-502. doi:10.1016/S0093-934X(03)00071-3

Cabeza, R., Ciaramelli, E., Olson, I. R., \& Moscovitch, M. (2008). The parietal cortex and episodic memory: An attentional account. $\mathrm{Na}$ ture Reviews Neuroscience, 9, 613-625. doi:10.1038/nrn2459

Ciaramelli, E., Grady, C. L., \& Moscovitch, M. (2008). Top-down and bottom-up attention to memory: A hypothesis (AtoM) on the role of the posterior parietal cortex in memory retrieval. Neuropsychologia, 46, 1828-1851. doi:10.1016/j.neuropsychologia.2008.03.022

Dobbins, I. G., Rice, H. J., Wagner, A. D., \& Schacter, D. L. (2003). Memory orientation and success: Separable neurocognitive components underlying episodic recognition. Neuropsychologia, 41, 318333. doi:10.1016/S0028-3932(02)00164-1 
Guerin, S. A., \& Miller, M. B. (2009). Lateralization of the parietal old/new effect: An event-related fMRI study comparing recognition memory for words and faces. NeuroImage, 44, 232-242. doi:10.1016/j .neuroimage.2008.08.035

Haramati, S., Soroker, N., Dudai, Y., \& Levy, D. A. (2008). The posterior parietal cortex in recognition memory: A neuropsychological study. Neuropsychologia, 46, 1756-1766. doi:10.1016/j .neuropsychologia.2007.11.015

Henson, R. N. A., Rugg, M. D., Shallice, T., \& Dolan, R. J. (2000). Confidence in recognition memory for words: Dissociating right prefrontal roles in episodic retrieval. Journal of Cognitive Neuroscience, 12, 913-923.

Klostermann, E. C., Kane, A. J. M., \& Shimamura, A. P. (2008), Parietal activation during retrieval of abstract and concrete auditory information. NeuroImage, 40, 896-901. doi:10.1016/j .neuroimage.2007.10.068

Noll, D. C., Stenger, V. A., Vazquez, A. L., \& Peltier, S. J. (1999). Spiral scanning in fMRI. In C. T. W. Moonen \& P. A. Bandettini (Eds.), Functional MRI (pp. 149-160). New York: Springer.

Paller, K. A., \& Wagner, A. D. (2002). Observing the transformation of experience into memory. Trends in Cognitive Sciences, 6, 93-102. doi:10.1016/S1364-6613(00)01845-3

RugG, M. D., \& Henson, R. N. A. (2002). Episodic memory retrieval: An (event-related) functional neuroimaging perspective. In A. E. Parker, E. L. Wilding, \& T. Bussey (Eds.), The cognitive neuroscience of memory encoding and retrieval (pp. 3-37). Hove, U.K.: Psychology Press.

SAMSON, S., \& ZatorRe, R. J. (1991). Recognition memory for text and melody of songs after unilateral temporal lobe lesion: Evidence for dual encoding. Journal of Experimental Psychology: Learning, Memory, \& Cognition, 17, 793-804.
Schloerscheidt, A. M., \& RugG, M. D. (1997). Recognition memory for words and pictures: An event-related potential study. NeuroReport, 8, 3281-3285.

Shannon, B. J., \& Buckner, R. L. (2004). Functional-anatomic correlates of memory retrieval that suggest nontraditional processing roles for multiple distinct regions within posterior parietal cortex. Journal of Neuroscience, 24, 10084-10092. doi:10.1523/ JNEUROSCI.2625-04.2004

Simons, J. S., Peers, P. V., Hwang, D. Y., Ally, B. A., Fletcher, P. C., $\&$ Budson, A. E. (2008). Is the parietal lobe necessary for recollection in humans? Neuropsychologia, 46, 1185-1191. doi:10.1016/j .neuropsychologia.2007.07.024

Smith, M. L. (1989). Memory disorders associated with temporallobe lesions. In F. Boller \& J. Grafman (Eds.), Handbook of neuropsychology (Vol. 3, pp. 91-106). Amsterdam: Elsevier.

UnCAPHeR, M. R., \& WAgner, A. D. (2009). Posterior parietal cortex and episodic encoding: Insights from fMRI subsequent memory effects and dual-attention theory. Neurobiology of Learning \& Memory, 91, 139-154. doi:10.1016/j.nlm.2008.10.011

VILberg, K. L., \& RUGG, M. D. (2008). Memory retrieval and the parietal cortex: A review of evidence from a dual-process perspective. Neuropsychologia, 46, 1787-1799. doi:10.1016/j.neuropsychologia.2008.01.004

Wagner, A. D., Shannon, B. J., Kahn, I., \& Buckner, R. L. (2005). Parietal lobe contributions to episodic memory retrieval. Trends in Cognitive Sciences, 9, 445-453. doi:10.1016/j.tics.2005.07.001

Zatorre, R. J., Belin, P., \& Penhune, V. B. (2002). Structure and function of auditory cortex: Music and speech. Trends in Cognitive Sciences, 6, 37-46. doi:10.1016/S1364-6613(00)01816-7

(Manuscript received March 6, 2009; revision accepted for publication May 12, 2009.) 